\title{
Deposition of Carbon Nanotube Films on Polyamide and Polypropylene Substrates: A Computer Simulation Approach
}

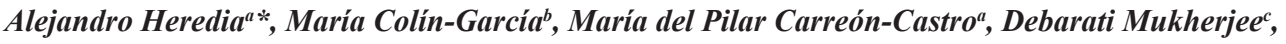

\author{
Bruno Abreu', David William Britt', Joana Catarina Mendes ${ }^{c}$
}

\author{
${ }^{a}$ Departamento de Química de Radiaciones y Radioquímica, Instituto de Ciencias Nucleares, \\ Universidad Nacional Autónoma de México, Circuito Exterior, Ciudad Universitaria, Apartado Postal \\ 70-543, C.P. 04510, D.F., México \\ ${ }^{b}$ Instituto de Geología, Universidad Nacional Autónoma de México, C.P. 04510, D.F., México \\ ${ }^{c}$ Centre for Mechanical Technology and Automation, Department of Mechanical Engineering, \\ University of Aveiro, 3810-193 Aveiro, Portugal \\ ${ }^{d}$ Biological Engineering, Utah State University, 84322-4105, Old Main Hill, Logan, Utah, USA
}

Received: January 25, 2016; Revised: April 22, 2016; Accepted: June 18, 2016

In this work we study hydroxylated carbon nanotube (CNT) assembly on polyamide (PA) and polypropylene (PP) polymers activated by UV radiation from a theoretical and experimental perspective. Molecular computer simulation was done to understand the stable conformations and bulk properties (molecular dynamics) of the polymers before and after exposure to UV radiation at the molecular level. Our experiments suggest that PA presents more - $\mathrm{OH}$ active groups, producing a more hydrophilic surface, whereas PP exhibits less potential UV activation. These results suggest that it is possible a facile covalent functionalization method to tune organic polymer surface properties through SWCNT anchoring for nanotechnological applications requiring defined surface roughness and chemical functionality on inexpensive polymers.

Keywords: Polypropylene, polyamide, SWCNT film, UV functionalization

\section{Introduction}

Carbon nanotubes (CNTs) exhibit a number of advantageous physical properties for many potential industrial and technical uses from different synthesis sources $^{1,2}$. For instance, they exhibit excellent thermal conductivity, mechanical strength ${ }^{2}$, optional semiconducting/metallic nature and advanced field-emission behavior ${ }^{3}$, that have been utilized in a number of different devices for several years ${ }^{4}$. In field emission behavior, which has been utilized for the stable anchoring of CNTs, is stressed as a route for new technological digital devices ${ }^{3}$. For this reason, thin films composed of CNTs and different polymers are an emerging class of materials with exceptional electrical, mechanical, and optical properties that can be readily integrated into many novel devices ${ }^{3-6}$. However, deposition of CNT films on polymers remains a technological challenge due to the inherent hydrophobicity of the polymer surfaces ${ }^{7-10}$ when water solutions of CNT derivatives are used. Another important aspect of hydrophobic polymers is that they readily bind proteins in the blood stream, limiting their use in vivo as stable implant materials ${ }^{11}$; this limitation presents a new path for hydrophilic surface modification through CNT derivatives. Many methods are common for anchoring of CNTs as dry surface techniques like corona discharge ${ }^{12}$, oxygen

*e-mail: aheredia@nucleares.unam.mx plasma $^{10}$, and UV radiation in the presence of ozone ${ }^{8}$. These methods effectively modify the surface properties of polymers, ceramics and glasses ${ }^{9}$.

The main effects of these treatments include cleaning (conversion of liquid films or solid contaminants into gaseous, volatile products), ablation/etching out of weak layers at the microscopic scale or even, selective etching of low molecular weight polymer chains and modification of chemical structures of surfaces through reactions of free radicals with surrounding gases $7,8,10,13-15$.

Out of these techniques, the $\mathrm{UV} / \mathrm{O}_{3}$ treatment/exposure is a convenient dry oxidizing method for activating the physicochemical features of polymer surfaces and other carbon materials ${ }^{14}$. It is a cost effective, environmentally friendly, rapid, and convenient technique 9 . This is primarily because the process can be conducted under atmospheric pressure, resulting in lower equipment and running costs. The individual and combined effects of UV light and ozone have been studied for various polymer surfaces, including styrene-butadine-styrene rubber $(\mathrm{SBS})^{16}$, polydimethylsiloxane (PDMS) $)^{8,17}$, polyethylene $(\mathrm{PE})^{18}$, polyethylene terephthalate $(\mathrm{PET})^{19}$, polypropylene $(\mathrm{PP})^{20}$, polyetheretherketone $(\mathrm{PEEK})^{21}$, as well as other surfaces. Here, we present an experimental and molecular modeling study for the anchoring of the CNT onto the organic polymers polyamide and polypropylene (Figure 1), which in addition to study the design of new surfaces, may lead to the design 


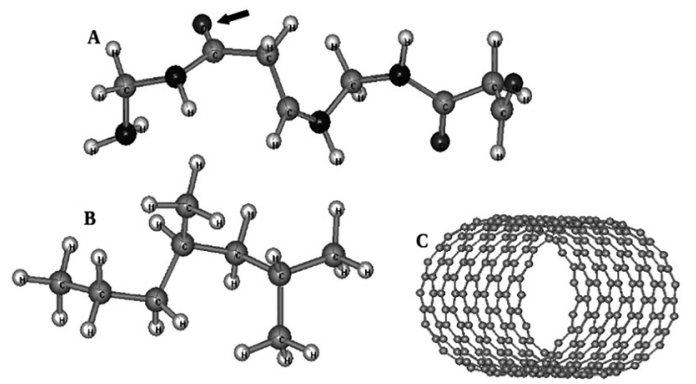

Figure 1: Polymers used as templates, PA (a) and PP (b), for the deposition of SWCNT (c). Some chemical groups such as the $\mathrm{C}=\mathrm{O}$ in polyamide (black arrow) might promote stability to the interaction with the SWCNT.

of sensors and CNT based composites ${ }^{22}$. Nowadays CNTs can be purchased form many different sources.

\section{Method}

The substrates used were polypropylene (CAS number 9003-07-0) and polyamide (CAS number 63428-84-2) polymers (Figure 1). Nanotubes selected were singlewalled CNTs (SWCNT were purchased from Cheap Tubes Inc, USA) with diameters ranging from 50 to 80 $\mathrm{nm}$, length ranging from 10 to $20 \mu \mathrm{m}$, with an aspect ratio from 222 to 400 , and a bulk density of $2.16 \mathrm{~g} / \mathrm{cm}^{3}$. The production of a suspension of SWCNT into a base fluid is not a straightforward process, as the SWCNT tend to agglomerate and settle with time. Thus, it was necessary to perform a chemical activation on the bulk SWCNT, based on the procedure described by Esumi et al., ${ }^{24}$ and applied previously to produce stable water suspensions of SWCNT. The suspension was prepared with $1 \mathrm{~g}$ of SWCNT in $40 \mathrm{ml}$ of a mixture of $\mathrm{HNO}_{3}$ and $\mathrm{H}_{2} \mathrm{SO}_{4}(1: 3$ volume ratio $)$ or $\mathrm{HNO}_{3}$ and refluxed at $140^{\circ} \mathrm{C}$. These were then washed with deionized water until the supernatant attained a $\mathrm{pH}$ of 7 . The cleaned SWCNT were then collected and dried in vacuum at $100^{\circ} \mathrm{C}$. The nano-fluids were prepared by ultrasonic mixing along with a magnetic stirrer, for 60 minutes in order to achieve a homogeneous suspension of SWCNT into the base fluids. The prepared nano-fluids were of different volume fractions of SWCNT $(0.25,0.5,0.75,1.0,1.5$ $\%$ ) in distilled water, ethylene glycol (EG) and distilled water (30\% EG, $60 \% \mathrm{EG})$. The effect of the exposure to the UV on the hydrophobicity of the surface of the polymers was measured using the contact angle method (Rame Heart model 100 Co., U.S.A) values of water on the polymer surfaces (Table 1). The sessile drop contact angle method was used for this characterization and the Laplace-Young method performed for calculation. For our study 3 spots were spread over the samples surface to obtain the average (Table 1).
Table 1: Contact angle of untreated and UV treated PA and PP polymers.

\begin{tabular}{lcc}
\hline Sample & $\begin{array}{c}\text { Exposure } \\
\text { duration }(\mathrm{hr} .)\end{array}$ & $\begin{array}{c}\text { Contact } \\
\text { angle }\left(^{\circ}\right)\end{array}$ \\
\hline PA (control) & 0 & 76.5 \\
PP (control) & 0 & 99.6 \\
PA & 1 & 36.9 \\
PP & 1 & 77.17 \\
PA & 2 & 46.79 \\
PP & 2 & 81.89 \\
PA & 3 & 36.05 \\
PP & 3 & 68 \\
PA (Sample stored in water) & 1 & 68.21 \\
PP (Sample stored in water) & 1 & 75 \\
\hline
\end{tabular}

\subsection{UV activation of the polymer substrates}

These polymers, due to their high content of C-H chemical groups (Figure 1), present a high hydrophobic nature. Since SWCNT suspensions are water-soluble, it is necessary to make the polymer surfaces hydrophilic by exposing them to UV radiation, in order to make them interact. Ultraviolet activation was performed with a mercury lamp (Pen ray UVP, UVC Light Sources, Model 11SC-2, 270 V, primary energy being $254 \mathrm{~nm}$, Cambridge, UK) over a period of 0 up to 3 hours (Figure 2) at a constant distance of $c a .2 \mathrm{~mm}$ from the surface of the polymers.

\subsection{Computer simulation by Molecular Mechanics $(\mathrm{MM+})$}

Molecular modeling studies were performed using the molecular mechanics method as implemented in the HyperChem program Version 8.0. (HyperCube, Canada $)^{25}$. Geometry optimizations were used to find the coordinates of molecular structures that represent a minimum potential energy. Full geometry optimization was performed using the HyperChem settings in the $\mathrm{MM}+$ force field, the Polak-Ribiere conjugate gradient algorithm and a root mean square gradient of 0.0001 $\mathrm{kcal} \cdot \AA^{-1} \cdot \mathrm{mol}^{-1}$. Molecular dynamics relaxation of the optimized structures was employed to look for possible local minima (step size of $0.001 \mathrm{ps,} \mathrm{constant} \mathrm{simulation}$ temperature of $300 \mathrm{~K}$ ). The organic molecules were geometrically optimized to determine the most stable conformation (MM+ method). In the case of the SWCNT it was obtained the most stable conformation and further, - $\mathrm{OH}$ groups added and optimized. The organic molecules, PA and PP, were chosen to interact with the SWCNT-OH (11.3 in diameter and 19.5 length, $120 \mathrm{OH}$ groups).

The optimized geometries obtained by the MM+ molecular mechanics method were further optimized 

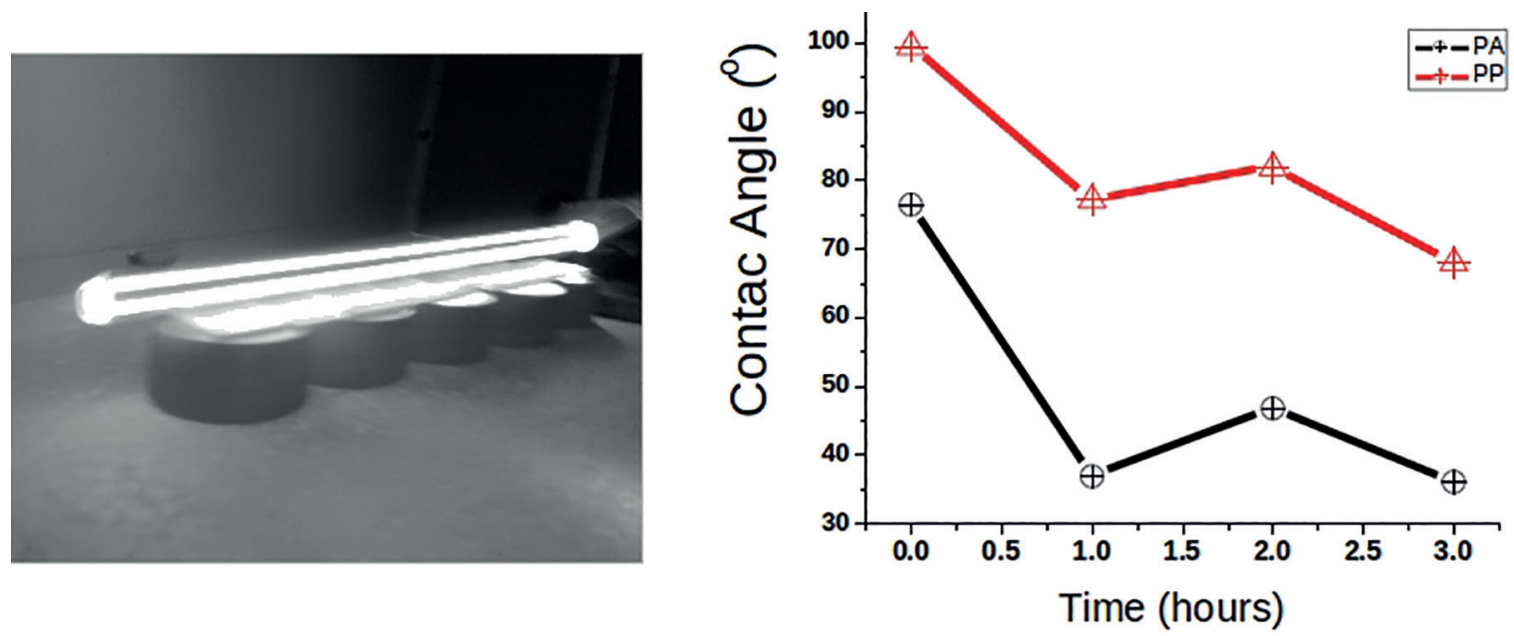

Figure 2: Polymers are put close to the UV lamp (left) and afterwards the contact angle measurements obtained (right) for $P P(\Delta)$ and $P A(O)$.

with PM3 semi-empirical methods by selecting the molecule and imported to a new work space. In semiempirical calculations, full geometry optimization were performed with the RHF basis, Polak-Ribiere conjugate gradient algorithm, and an RMS gradient of 0.0001 $\mathrm{kcal} \cdot \AA^{-1} \cdot \mathrm{mol}^{-1}$ as recommended in the HyperChem manual and mentioned previously as the most suitable method for organic molecules.

\section{Results and discussion}

Experimental results of the PA with SWCNT (Figure 2 right) show lower contact angles, what preliminary agrees with the molecular model with PA interacting to the SWCNT.

\subsection{Computer simulation}

In order to understand the possible interactions between chemical groups from individual PA and PP molecules due to docking with the SWCNT, we have performed $\mathrm{MM}+$ simulations (Table 2) in gas phase and with water molecules. Ultraviolet functionalization with $\mathrm{NH}_{3}$ and $\mathrm{O}_{2}$ of carbon nanotubes produce $\mathrm{NH}_{2}$ and -OH groups which in turn might yield covalent bonds with other organic compunds ${ }^{26}$. For this reason, we included in our models the SWCNTs with OH chemical groups. By combining geometrical optimization and molecular dynamics for establishing relaxation of the optimized structures, we determined a number of local minima energy for every molecule, evaluated at different conformations. The most stable structure that was obtained for our models is the PA in water, with an energy of $-1230 \mathrm{kcal} \mathrm{mol}^{-1}$. In this conformation, it was possible for amide groups to approach to the regions that are rich in $-\mathrm{OH}$ in the SWCNT (Figure 3C).
Table 2: Local energy minima for the different molecular conformations.

\begin{tabular}{lll}
\hline & Energies $(\mathrm{kcal} / \mathrm{mol})$ & Closest distance $(\AA)$ \\
\hline SWCNT & -128 & \\
SWCNT-OH & 6479 & \\
PA+SWCNT & 30.2 & 2.6 \\
PP+SWCNT & 4 & 2.9 \\
SWCNT+H2O & -1128 & \\
PA+SWCNT+H2O & -1230 & \\
PP+SWCNT+H2O & -1122 & \\
\hline
\end{tabular}

A
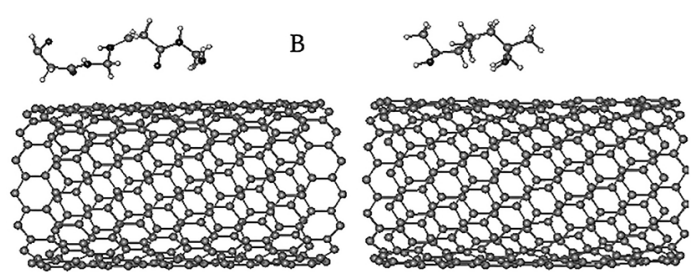

$\mathrm{C}$

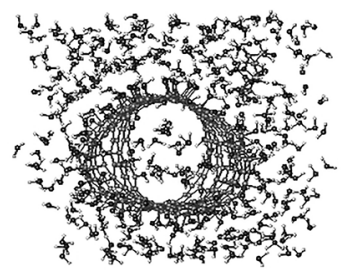

Figure 3: Models of the (A) SWCNT and PA, (B) SWCNT and PP and $(\mathrm{C})$ the model of the SWCNT functionalized with $-\mathrm{OH}$ groups in a water box. The PA is closer to the SWCN than the PP polymer model (see closest distances in Table 2).

In the molecular dynamics results (Figure 4. Ekin, Temp, Etot and Epot corresponds to kinetic energy, temperature, total energy and potential energy respectively), water molecules bring a reduction in total energy (Figure 4 A,B ca. $250 \mathrm{kcal} / \mathrm{mol}$ vs $c a$. $0 \mathrm{kcal} / \mathrm{mol}$ ) the PP fits in well with the experimental contact angle results as more hydrophobic (Figure 2 and 4 C,D). 

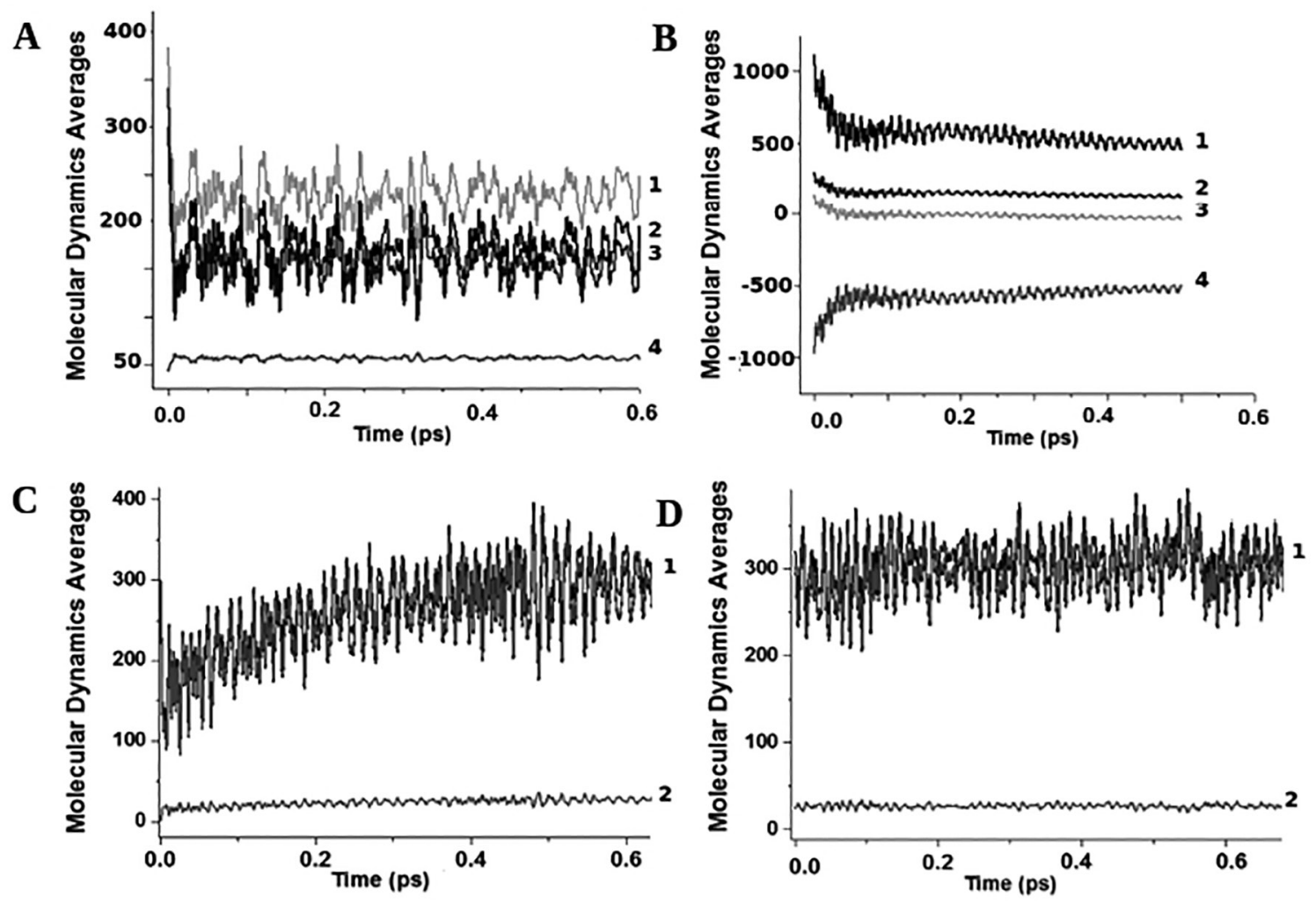

Figure 4: Molecular dynamics of the PA (A, B) and PP (C, D) polymers (energies in $\mathrm{kcal} / \mathrm{mol}$ and temperature in $\mathrm{K})$. The simulation results in PA show a significant reduction in (A) total and potential energy before (1 total energy, 2 kinetic energy, 3 temperature, 4 potential energy) and (B) after its incorporation in water molecules (1 kinetic energy, 2 temperature, 3 total energy 4 potential energy) whereas in (C) PP with no water molecules and (D) after combination with water molecules the total and kinetic energies and temperatures (1) are very similar, excepting potential energy (1).

The polyamide is active in the infrared in its chemical groups $(\mathrm{C}=\mathrm{O}, \mathrm{NH}$ and $\mathrm{C}-\mathrm{H})$ and we have also identified the infrared spectrum of the so called "amide" group corresponding to two bands at 1650 and $1550 \mathrm{~cm}^{-1} 27$. Albeit the polyamide identified bands are shifted in the PM3 simulations (the ones at 1981 and $1919 \mathrm{~cm}^{-1}$ ) (Figure 5) we implemented a correction factor already used for PM3 semi-empirical method ${ }^{28}$. We performed a correction of the frequencies by a factor is 0.82 . This is $1981(0.82)=1624 \mathrm{~cm}^{-1}$ and $1919(0.82)=1573 \mathrm{~cm}^{-1}$ it means a shift of almost the $20 \%$ of the experimental IR amide bands. A similar procedure was performed on the polypropylene PM3 FTIR simulation (with a correction factor of 1.005 for the band at $2836 \mathrm{~cm}^{-1}$ and 0.95 for the band at $3069 \mathrm{~cm}^{-1}$ ). Here, some difficulties must be overthrown to identify the specific $\mathrm{C}$-H-related chemical groups by regions. The reason of this difficulty, is the homogeneous (CH-based) chemical structure of the polypropylene distinctively at the $c a .3000$ to 2800 $\mathrm{cm}^{-1}$ and $\mathrm{ca} .900$ to $600 \mathrm{~cm}^{-1}$ regions ${ }^{29}$ (Figure 5).

\section{Conclusions}

Here is shown a possible way to align SWCNT to obtain a carbon nanotube high surface density incorporated in the surface of polymer slabs. This proposal opens the way to implement additional steps to the mechanically assisted assembled polymers ${ }^{17}$. The results indicate that the simulated UV irradiation might be useful in increasing the hydrophilicity of polyamide and polypropylene polymers. The experimental method presented here proposes a potential functionalization of organic polymers with high performance to further control the surface roughness through SWCNT anchored for nanotechnological applications. The theoretical response of polyamide and polypropylene polymers with SWCNT was investigated. The lowest energies (more stability) in the PA model with one molecule of polyamide interacting with one SWCNT shows the more hydrophilic behavior than in polypropylene after $-\mathrm{OH}$ chemical adjustment. The infrared spectra are not giving the complete, accurate information perhaps to a deficient simulation time. The correction factor for the IR bands, shows a clearer inconsistency in the experimental FTIR from amide I and II bands from the polyamide than in the simulated polypropylene, although a more accurate study might give a more correct infrared signal of the PA and PP polymers. In our group, we consider activating PA and PP to anchor functionalized CNT through different methods. 


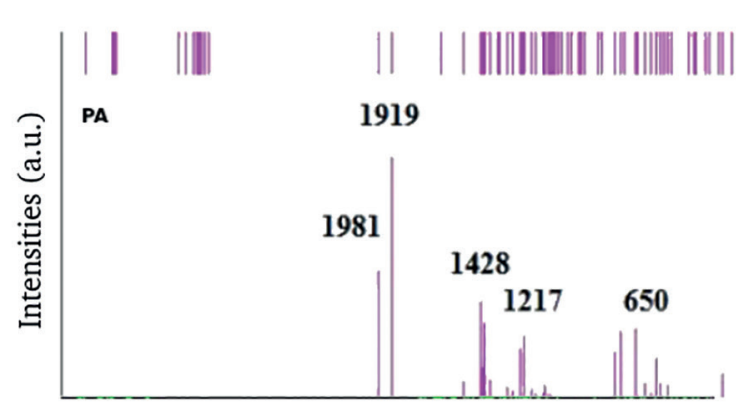

Wavenumber $\left(\mathrm{cm}^{-1}\right)$

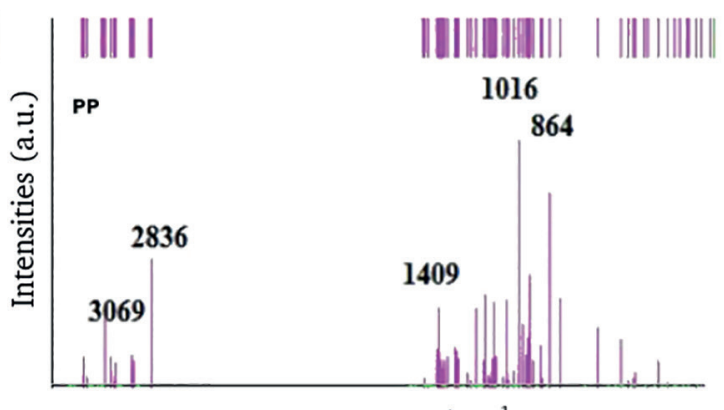

Wavenumber $\left(\mathrm{cm}^{-1}\right)$

Figure 5: Simulated infrared spectra of (A) polyamide and (B) polypropylene. Corrections in the spectra are performed to identify the experimental bands.

Our results here show a preliminary improvement in binding SWCNT to organic polymers that in turn will be useful for aligning CNT to obtain a high surface density of bioactive CNT.

\section{Acknowledgements}

J.C. Mendes acknowledges FCT through POPH/ FSE (Programa Operacional Potencial Humano/Fundo Social Europeu) for the grant SFRH/BPD/90306/2012 with FEDER/COMPETE funds. We thank a lot the thechnical support for the computer simulations to M.Sc. Luciano Díaz González, Mr. Martín Cruz Villafañe and Eng. Eduardo Murrieta León.

\section{References}

1. Mendoza D, Santiago P, Reyes Pérez E. Carbon nanotubes produced from hexane and ethanol. Revista Mexicana de Física. 2006;52(1):1-5.

2. Ruoff RS, Lorents DC. Mechanical and thermal properties of carbon nanotubes. Carbon. 1995;33(7):925-930.

3. Milne WI, Teo KBK, Amaratunga GAJ, Legagneux P, Legagneux L, Schnell JP, et al. Carbon nanotubes as field emission sources. Journal of Materials Chemistry. 2004;14(6):933-943.

4. Connolly T, Smith RC, Hernandez Y, Gun'ko Y, Coleman JN, Carey JD. Carbon-nanotube-polymer nanocomposites for fieldemission cathodes. Small. 2009;5(7):826-831.

5. Cao A, Veedu VP, Li X, Yao Z, Ghasemi-Nejhad MN, Ajayan PM. Multifunctional brushes made from carbon nanotubes. Nature Materials. 2005;4:540-545.

6. Ramakrishna S, Mayer J, Wintermantel E, Leong KW. Biomedical applications of polymer-composite materials: a review. Composites Science and Technology. 2001;61(9):1189-1224.

7. Øiseth SK, Krozer A, Lausmaa J, Kasemo B. Ultraviolet light treatment of thin high-density polyethylene films monitored with a quartz crystal microbalance. Journal of Applied Polymer Science. 2004;92(5):2833-2839.

8. Efimenko K, Wallace WE, Genzer J. Surface modification of Sylgard-184 poly(dimethyl siloxane) networks by ultraviolet and ultraviolet/ozone treatment. Journal of Colloid and Interface Science. 2002;254(2):306-315.
9. Mittal KL, Pizzi A. Adhesion Promotion Techniques: Technological Applications. Boca Raton: CRC Press; 1999.

10. Strobel M, Lyons CS, Mittal KL. Plasma Surface Modification of Polymers: Relevance to Adhesion. Boca Raton: CRC Press; 1994.

11. Chumbimuni-Torres KY, Coronado RE, Mfuh AM, CastroGuerrero C, Silva MF, Negrete GR. Adsorption of Proteins to Thin-Films of PDMS and Its Effect on the Adhesion of Human Endothelial Cells. RSC Advances. 2011;1(4):706-714.

12. Sun C, Zhang D, Wadsworth LC. Corona treatment of polyolefin films-A review. Advances in Polymer Technology. 1999;18(2):171-180.

13. Childs WR, Motala MJ, Lee KJ, Nuzzo RG. Masterless Soft Lithography: Patterning UV/Ozone-Induced Adhesion on Poly(dimethylsiloxane) Surfaces. Langmuir. 2005;21(22):1009610105.

14. MacManus LF, Walzak MJ, McIntyre NS. Study of ultraviolet light and ozone surface modification of polypropylene. Journal of Polymer Science Part A: Polymer Chemistry. 1999;37(14):2489-2501.

15. Sham ML, Li J, Ma PC, Kim JK. Cleaning and Functionalization of Polymer Surfaces and Nanoscale Carbon Fillers by UV/ Ozone Treatment: A Review. Journal of Composite Materials. 2009;43(14):1537-1564.

16. Romero-Sánchez MD, Pastor-Blas MM, Martín-Martínez JM, Walzak MJ. UV treatment of synthetic styrene-butadienestyrene rubber. Journal of Adhesion Science and Technology. 2003;17(1):25-45.

17. Genzer J, Efimenko K. Creating long-lived superhydrophobic polymer surfaces through mechanically assembled monolayers. Science. 2000;290(5499):2130-2133.

18. Martínez-Romo A, González Mota R, Soto Bernal JJ, Frausto Reyes C, Rosales Candelas I. Effect of ultraviolet radiation in the photo-oxidation of High Density Polyethylene and Biodegradable Polyethylene films. Journal of Physics: Conference Series. 1015;582:012026.

19. Walzak MJ, Flynn S, Foerch R, Hill JM, Karbashewski E, Lin A, et al. UV and ozone treatment of polypropylene and poly(ethylene terephthalate). Journal of Adhesion Science and Technology. 1995;9(9):1229-1248.

20. Rabello MS, White JR. Polypropylene photodegradation: An inhomogeneous process. Polímeros. 1997;7(2):47-57.

21. Mylläri V, Ruoko TP, Järvelä P. The effects of UV irradiation to polyetheretherketone fibres - Characterization by different techniques. Polymer Degradation and Stability. 2014;109:278-284.

22. Spitalsky Z, Tasis D, Papagelis K, Galiotis C. Carbon nanotubepolymer composites: Chemistry, processing, mechanical and electrical properties. Progress in Polymer Science. 2010;35(3):357-401. 
23. Basiuk VA, Basiuk EV, eds. Chemistry of carbon nanotubes. Stevenson Ranch: American Scientific Publishers; 2008.

24. Esumi K, Ishigami M, Nakajima A, Sawada K, Honda H. Chemical treatment of carbon nanotubes. Carbon. 1996;34(2):279-281.

25. Hypercube. HyperChem Professional 8.0. [cited 2016 Jun 22]. Available from: http://www.hyper.com/?tabid=360

26. Girard-Lauriault PL, Illgen R, Ruiz JC, Wertheimer MR, Unger WES. Surface functionalization of graphite and carbon nanotubes by vacuum-ultraviolet photochemical reactions. Applied Surface Science. 2012;258(22):8448-8454.
27. Miyake A. Infrared spectra and crystal structures of polyamides. Journal of Polymer Science Part B: Polymer Physics. 1960;44(143):223-232.

28. Lubes G, Gómez G, Hernández L, Carpio ED, Lubes V. Theoretical Studies of(E)-2-[(2-Chloro-benzylimino)-Methyl]-Methoxyphenol, a Schiff base. Avances en Química. 2014;9(1):29-36.

29. Longo C, Savaris M, Zeni M, Brandalise RN, Grisa AMC. Degradation study of polypropylene (PP) and bioriented polypropylene (BOPP) in the environment. Materials Research. 2011;14(4):442-448. 